\title{
Effects of vertical and horizontal plyometric exercises on explosive capacity and kinetic variables in professional long jump athletes
}

Amir Vazini Taher ${ }^{1 \mathrm{ABCD}}$, Ratko Pavlovic ${ }^{2 \mathrm{BCDE}}$, Shahram Ahanjan ${ }^{3 \mathrm{ABCE}}$, Iryna Skrypchenko ${ }^{4 \mathrm{ABDE}}$, Marko Joksimovic ${ }^{5 \mathrm{ABD}}$

${ }^{1}$ Education Administration, Physical Education and Sport Sciences, Iran

${ }^{2}$ University of East Sarajevo, Bosnia and Herzegovina

${ }^{3}$ Amirkabir University of Technology, Iran

${ }^{4}$ Dnipropetrovs 'k State University of Internal Affairs, Ukraine

${ }^{5}$ Football Club National, Montenegro

Authors' Contribution: A - Study design; B - Data collection; C - Statistical analysis; D - Manuscript Preparation; E - Funds Collection

$\begin{array}{ll}\text { Abstract } & \text { Athletic jumps are specific cyclically-acyclic movements that despite the good performance of the } \\ \text { Purpose: } & \text { techniques require from competitors a high level of motor, specific-motor and functional abilities. The } \\ & \text { aim of this study was to examine the response effect of vertical and horizontal plyometric training on } \\ & \text { explosive capacity and kinetic variables in long jump athletes. } \\ & \text { The participants of this study were twenty professional jumpers (22.5 } \pm 4.2 \text { years; } 178.4 \pm 9.8 \mathrm{~cm} ; 70.3 \\ & \pm 7.6 \mathrm{~kg}) \text { who were divided into two groups: experimental (plyometric training) and control (standard } \\ \text { Material: } & \text { training). They participated in the last track and field championship in country, moreover, three of } \\ & \text { them participated in the last Asian games, and one athlete participated in the world track and field } \\ & \text { championship. The experiments were conducted on June-July } 2019 \text { in twenty professional athletes. All } \\ & \text { tests were performed after a standard warm up protocol. The place of camera was always determined } \\ & \text { wisely around the jumping field to attain best photography. Organizing and controlling the imaging and } \\ & \text { motor analysis processes were done by a biomechanics expert. } \\ & \text { Post training results in experimental group showed more improvement in } 30 \mathrm{~m} \text { sprint, vertical jump, } \\ & \text { horizontal velocity at take-off, and long jump completion, comparing the control group. Significant } \\ \text { Results: } & \text { between group differences in all variables were detected post training. No significant post training } \\ & \text { improvements in flight time and take off duration were reported in control group. } \\ \text { Conclusions: } & \text { Vertical and horizontal plyometric training protocol was shown to be more effective in promoting } \\ & \text { improvement in explosive capacity than kinetic variables. } \\ \text { Keywords: } & \text { deep jump, plyometric, long jump, vertical and horizontal exercises }\end{array}$

\section{Introduction}

Athletic jumps are specific cyclically-acyclic movements that despite the good performance of the techniques require from competitors a high level of motor, specific-motor and functional abilities. Also, all the jumping disciplines contain appropriate morphological profile of athletes (height, weight, BMI, age) that is characteristic for it. It is usually said that the jumpers are of high growth and relatively low weight, with long legs, long and thin muscles, and the muscle structure is dominated by white muscle fibers [1]. The long jump is an athletic discipline of speed-strong character and with triple jump it belongs to a group of remote jumps, in relation to the trajectory of the body center it belongs to the horizontal jumps $[2,3]$. The speed of running start is as important as the strength of the lower extremities which give the final impetus bounce, so the result depends on the speed, jumping ability and technique of movement [4]. In top-level long jump athletes, capacity to quickly produce and apply a significant sum of force is plays a basic role [5]. It appears that the capability to arrange the resultant force vector horizontally while accelerating may

\footnotetext{
(c) Amir Vazini Taher, Ratko Pavlovic, Shahram Ahanjan,

Iryna Skrypchenko, Marko Joksimovic, 2021
}

doi:10.15561/26649837.2021.0205 be a key determinant of speed [6]. Subsequently, athletes who are able to create enormous amounts of forces onto the ground within the forward course (i.e., horizontal plane) are more likely possible to attain more amounts of speeds while accelerating [7]. In contrast, it has been appeared that the switch from lower to higher speeds attains in shorter support stage term with concomitant increases in vertical peak force [8]. Long jump technique is based on a natural and quite easy movement, where the jumper strives for greater speed (horizontal componenthorizontal shot) which will convert the reflection to the greater distance jump (ballistic curve-pitched shot). The ratio of the horizontal component (speed of running start) and vertical component (speed of reflection, flash) is in relation 2:1. The effect of the horizontal and vertical components directs the body so that an elevation angle can be from $18^{\circ}-26^{\circ}$. This means that the decreasing of the angle $(\beta)$ increases a result of movements $(R)$ reducing the elevation angle $(\alpha)$. Bearing all this in mind, a reflection with the long jump should be executed at top speed and to the limit only after the moment of verticals. Research of some authors have confirmed the inverse relationship of horizontal and vertical body centre ascent, ie. with increasing horizontal speed decreases the vertical and 
vice versa $[9,10]$. Many sports movements depend on the high achievements gained in specific technical stages, then it should be taken care of and developed to the top level and possible level, for example in the jumping movements into track and field sports the approaching stage is the main stage and planned for achievement, while in the running and throwing movements into track and field games the stage of throwing or running is the most important stage in these respects or events [11]. In all jumps there is an unwritten rule that every next stage in the technique of execution is conditioned by the previously performed step (accuracy of movement). Any mistake made has a significant impact on the accuracy of movement in the coming stages and the final result of competitors [1]. The force-vector hypothesis has been proposed to guide coaches and researchers in selecting the most appropriate exercises and drills for each particular stage of running. For case, Contreras et al. confirmed that the horizontally-oriented hip-thrust is predominant to the front squat to extend increasing speed over $20-\mathrm{m}$ after a short-term period (i.e., 6-week), which is possibly related to the anteroposterior constrain vector utilized in this process and can affect even motivation generation [12]. On the other hand, Kale et al. illustrated that, among a few variables collected in vertical and horizontal bounced, the drop hop stature is the most excellent pointer of speed accomplished by a tip top sprinter all through a $100-\mathrm{m}$ sprint race [13]. Comparable comes about were gotten in an arrangement of later examinations executed with sprinters and competitors, affirming that the heading of the resistance constrain vector relative to the body is determinant in intervening adjustments to speed qualities [14]. Be that as it may, in spite of the fact that a few of these considers have been carried out utilizing vertical works out performed at the ideal control zones (i.e., utilizing loads able of maximizing control yield) [8], there's a need of investigating this subject with horizontally-directed works outs (e.g., hip-thrust).The long jump consists of 4 phases: run phase, take-off phase, flight phase, and landing phase [15]. Long jump performance depends on not only a fast horizontal velocity at the end of the runup but also take-off technique and landing in the sandy area. Successful long jump athletes take a good care of these stages because they affect the distance of long jump, especially, the run phase which is the first phase of the long jump [15]. Each of these phases has its cinematic specificity in terms of performance, which requires full attention and concentration from the competitor. Running start is the first phase in the structure of jumps, which should provide a good starting speed of body flight (Vo), respectively, to achieve the best transition of the maximum speed in the best reflection of jumpers [4]. Precisely in this combination and transition of maximum speed in the final steps and reflection there is a part of the biggest secrets of the success of top jumpers. Many jumpers use their maximal speed of approach combined with technique (optimal technique is used to achieve as bigger speed while sprinting as possible and to bounce off as much as possible) hoping to achieve the longest possible distance
[16]. Most of the speed and strength training exercises that jump trainers recommend through their training programs depend on weight. While some exercises do not match the level of skill capability it seems from previous researches that the use of different deep jump methods (horizontal - vertical) for the purpose of developing the physical capacity and biomechanical characteristics is effective for athletes [17]. The present study provides a period of horizontal and vertical plyometric exercises (Drop Jumps) in the development of explosive capacity and kinetic variables in professional long jump athletes.

\section{Materials and Methods}

\section{Participants}

The participants of this study were twenty professional jumpers $(22.5 \pm 4.2$ years; $178.4 \pm 9.8 \mathrm{~cm} ; 70.3 \pm 7.6$ $\mathrm{kg}$ ) who were divided into two groups: experimental (plyometric training) and control (standard training). They participated in the last track and field championship in country, moreover, three of them participated in the last Asian games, and one athlete participated in the world track and field championship. Prior to the experimental phase of study, athletes were briefed on the experimental design and signed an informed consent form.

\section{Study design}

In this randomized controlled trial, the long jump athletes in experimental and control groups performed pretest and posttest examinations to determine the effects of vertical and horizontal plyometric exercises on explosive capacity and kinetic variables. The athletes in experimental group were guided to perform plyometric drills as following: Box Jumps, Hurdle hops, Double leg "X" hop, Backward Throw, Ankle jumps, Ankle Hops, Power Skips, Lateral Taps on a Ball, Jump and turn $180^{\circ}$, Standing Jump \& Reach, Split squat jump, Single leg zig-zag drill. The participants of control group were training under a standard training protocol same as the experimental group but with different drills. Some of the drills they performed were: Full speed 30-50 meter, Landing drills, Five stride jumps into pit, Take off drills, Long jump approaches 4-8, Conditioning Circuits, Timing and coordination, Pick on additional take off. In table 1 shows is pre training physical characteristics (table 1).

\section{Measurements}

Means, tools and devices used for this study are mentioned as follows:

- Electronic stopwatch;

- Computer;

- Metal measurement tape;

- Wooden shutters with different heights;

- Different height barriers and boxes;

- Long jump field;

- Camera and stand.

The researchers prepared explosive capacity and kinetic variables tests as follows:

- 30 m sprint;

- Vertical jump;

- Standing long jump;

- Max vertical height; 
- Horizontal velocity at take off;

- Vertical velocity at take off;

- Flight time;

- Take off duration;

- Long jump completion.

Procedure

The experiments were conducted on June-July 2019 in twenty professional athletes. All tests were performed after a standard warm up protocol. The place of camera was always determined wisely around the jumping field to attain best photography. Organizing and controlling the imaging and motor analysis processes were done by a biomechanics expert.

Training protocol

The training program aimed to develop the explosive capacity of the athletes using the various jumps as one of the modern methods used for the development of this capacity. The exercises were performed at full speed, to ensure that the endurance factor does not interfere with the muscular ability. $60 \%$ to $80 \%$ of the maximum capacity of the athlete were chosen for horizontal and vertical jumps. The number of athletes in each group for warm up and testing were 2-3 and the number of repetitions were from 6 to 12 times with an active rest ( 3 min between sets and 4090 seconds between repetitions). The whole program lasted between 50-60 minutes for each athlete.

Statistical Analysis

IBM SPSS statistics version 22.0 package was used to analyse data. The descriptive data is reported as means \pm standard deviations and percentage of changes. Physical characteristics before the intervention were tested for difference between the groups with unpaired t-tests. Test data was examined for normality with Shapiro Wilk test and for homogeneity of variances with Levene's test. If normality of data was present a two way mixed analysis of variance (factors: group, time, and interaction (group vs. time)) was performed. Effect sizes estimates are given in terms of partial eta-squared measures $(\eta 2 p)$. where a significant interaction effect was seen, post-hoc t-tests were applied to decide the source of difference. The level of significance for all measures considered at $\mathrm{p}<0.05$.

\section{Results}

At baseline there was no significant difference between two groups in any of the measured variables. Test data was normally distributed in 7 out of the 9 tests table 2 . A mixed $2 * 2$ multi-factorial ANOVA indicated a significant interaction effect for $30 \mathrm{~m}$ sprint $(\mathrm{F}=54, \mathrm{p}=$ $\left.\left..00, \mathrm{n}^{2} \mathrm{p}=.66\right)\right)$, vertical jump $\left.\left(\mathrm{F}=12, \mathrm{p}=.00, \mathrm{n}^{2} \mathrm{p}=.31\right)\right)$, standing long jump $\left.\left(\mathrm{F}=38, \mathrm{p}=.00, \mathrm{n}^{2} \mathrm{p}=.58\right)\right)$, max vertical height (NA), Horizontal velocity at take-off $(\mathrm{F}=70, \mathrm{p}=$ $\left..00, \mathrm{n}^{2} \mathrm{p}=.72\right)$ ), flight time $\left.\left(\mathrm{F}=24, \mathrm{p}=.00, \mathrm{n}^{2} \mathrm{p}=.47\right)\right)$, take-off duration $\left.\left(\mathrm{F}=15, \mathrm{p}=.00, \mathrm{n}^{2} \mathrm{p}=.34\right)\right)$, long jump completion (NA). No significant interaction effect was found for vertical velocity at take-off $(F=30, p=.01$, $\left.\mathrm{n}^{2} \mathrm{p}=.56\right)$ ). Further analyses with paired t-tests showed significant within group differences between pre- and post-tests in both groups for all variables. Effects sizes varied from $d=0.4$ to 6.5 . Changes in the experimental group were significantly greater compared to the control group for $30 \mathrm{~m}$ sprint, vertical jump, horizontal velocity at take-off, and long jump completion.

Table 1. Pre training physical characteristics

\begin{tabular}{lllll}
\hline Basic Variables & Measuring unit & SMA & $\begin{array}{l}\text { Standard } \\
\text { deviation }\end{array}$ & $\begin{array}{l}\text { Skewness } \\
\text { coefficient }\end{array}$ \\
\hline Age & Years & 22.5 & 4.2 & 0.39 \\
Height & Centimetre & 178.4 & 9.8 & 0.38 \\
Weight & Kg & 70.3 & 7.6 & 0.42 \\
BMI & Kg.m & 22.17 & 5.4 & 0.75 \\
Experience & Years & 5.7 & 2.3 & 0.48 \\
\hline
\end{tabular}

Table 2. The results of explosive capacity and kinetic variables examinations

\begin{tabular}{|c|c|c|c|c|c|c|c|}
\hline \multirow[b]{2}{*}{ Variables } & \multicolumn{2}{|c|}{ Experimental group } & \multicolumn{2}{|c|}{ Control group } & \multirow{2}{*}{$\begin{array}{l}\text { P-value } \\
\text { (interaction } \\
\text { effect) }\end{array}$} & \multicolumn{2}{|l|}{ Value (d)-p } \\
\hline & pre & post & pre & post & & experimental & control \\
\hline $30 m$ sprint & $3.74 \pm 0.23$ & $3.49 \pm 0.18$ & $3.76 \pm 0.21$ & $3.62 \pm 0.20$ & $0.00(0.66)$ & $0.00 *(2.4)$ & $0.00 *(1.5)$ \\
\hline Vertical jump & $39.21 \pm 2.08$ & $51.48 \pm 6.12$ & $39.37 \pm 2.43$ & $46.75 \pm 3.32$ & $0.00(0.31)$ & $0.00 *(2.3)$ & $0.00 *(2.6)$ \\
\hline Standing long jump & $230 \pm 5.82$ & $251 \pm 9.74$ & $231 \pm 4.23$ & $238 \pm 5.71$ & $0.00(0.58)$ & $0.00 *(2.3)$ & $0.00 *(1.9)$ \\
\hline Max vertical height & $114 \pm 4.56$ & $146 \pm 5.35$ & $115 \pm 5.12$ & $121 \pm 6.38$ & $0.00(0.63)$ & $0.00 *(6.5)$ & $0.00 *(1.3)$ \\
\hline $\begin{array}{l}\text { Horizontal velocity at } \\
\text { take off }\end{array}$ & $7.95 \pm 0.32$ & $8.89 \pm 0.51$ & $7.64 \pm 0.39$ & $7.87 \pm 0.30$ & $0.00(0.72)$ & $0.00 *(2.1)$ & $\begin{array}{l}0.0 *^{*} \\
(0.58)\end{array}$ \\
\hline $\begin{array}{l}\text { Vertical velocity at } \\
\text { take off }\end{array}$ & $2.98 \pm 0.50$ & $3.06 \pm 0.77$ & $2.94 \pm 0.13$ & $2.99 \pm 0.34$ & $0.56(0.01)$ & $0.00 *(0.7)$ & $0.00 *(0.4)$ \\
\hline Flight time & $0.65 \pm 0.36$ & $0.73 \pm 0.03$ & $0.64 \pm 0.33$ & $0.66 \pm 0.02$ & $0.00(0.47)$ & $0.00 *(1.7)$ & $0.63(1.2)$ \\
\hline Take off duration & $0.21 \pm 0.02$ & $0.18 \pm 0.01$ & $0.20 \pm 0.01$ & $0.19 \pm 0.02$ & $0.00(0.34)$ & $0.00 *(1.8)$ & $0.61(1.1)$ \\
\hline $\begin{array}{l}\text { Long jump } \\
\text { completion }\end{array}$ & $5.91 \pm 0.10$ & $6.48 \pm 0.20$ & $5.86 \pm 0.11$ & $6.19 \pm 0.15$ & $0.00(0.48)$ & $0.00 *(3.9)$ & $0.00 *(2.2)$ \\
\hline
\end{tabular}




\section{Discussion}

The findings of study in both groups indicated that jump performance had improvements after 8 weeks vertical and horizontal plyometric exercises. The experimental group outperformed in 7 of the 9 parameters when compared to the traditional training program. Our training method (deep jump exercises) which bear a resemblance to the long jump take off, comprise stretch-shortening cycles of different muscles in lower extremity. This method appeared to be favorable for enhancing sprint and jump performance, long jump distance and some other related parameters [18]. This could be due to the fact that deep jump exercises help the athletes to learn the compatibility between arms and legs movement.

The findings of this study in line with previous researches revealed that vertical and horizontal plyometric exercises have a significant effect on long jump performance $[19,20]$ and also significantly enhances the hip and thigh power which was projected by vertical jump test in this study [21, 22]. Furthermore, our results are in line with the findings of Hewett et al. [23] who indicated an improved hamstring muscle peak power, torque, and hamstring/quadriceps ratio after a period of plyometric jump training.

Plyometric training might have other enhancing effects in addition to performance enhancement, such as knee joint motion stabilization by increasing the lower extremity strength. Simek-Salaj, Milanovic, Jukic [24] determined the changes in agility and explosive strength, the type of jumping ability under the influence of a proprioceptive training program on a sample of physical education students. It was determined that, in the case of untrained participants, changes in vertical jumping ability are possible under the influence of proprioceptive training, and in the case of trained participants, the effects were smaller or not significant.

Markovic et al. [25] studied the results of sprint and plyometric training on the morphological characteristics of young, physically active participants. The research showed that these two methods have a limited effect on the morphological dimensions of younger men. The efficiency of technique depends on the skill of jumper to exercise great pressure on the track in the small protrude of leg, especially in the last step, which provides the necessary height of the jump and the horizontal movement of the body.

Thus excellent jumpers, depending on gender, develop great pressure force (from $300-400 \mathrm{~kg}$ ) on the board, where the stepping leg slightly bents at the knee $\beta=$ $175^{\circ}-178^{\circ}$ or $165-172^{\circ}$ [9], slightly less at the hip joint $(\gamma$ $\left.=165^{\circ}-170^{\circ}\right)$, and there is also a partially bending at the joints of the spine. It all causes eccentric character of the work. Intentional loosening of the knee joint is done in order to utilize the forces of mm. quadriceps femoris to the extent where leg can withstand the pressure, because it is not possible to avoid the torque of the pressure component in relation to the knee joint. In order to offset the effect of this moment, and to achieve the rebound it is necessary that the product of muscle force $(\mathrm{Q})$ and its prong ( $\mathrm{r}$ is constant) is equal to or greater than the product of the pressure force $(\mathrm{R})$ and its prong $(\mathrm{k})$. The current top jumpers at the time of the rebound have less flexion of the knee joint but a stronger force of quadriceps [1]. The deep jump (vertical - horizontal) exercises were beneficial in the development of the explosive power of long jump athletes. These training exercises help to develop the high coordination of the neuro muscular system to respond more strongly and faster during the performance of complicated sport related movements.

Sharkey [26] points out that the various jump exercises increase the explosive capacity of athletes, especially in the jumping competitions. In view of that, the results of our research imply that the deep jump training is a powerful and effective way to improve the strength and speed in long jump athletes, allowing the nervous system to activate the largest number of muscle fibers. Thus, in athletes who need to integrate the maximum strength of muscles with the maximum speed in performing sport movements, especially if the explosive capacity is one of the qualities required, vertical and horizontal plyometric exercises are useful.

Less et al. [9] an attempt was made to identify the mechanism acting during the touchdown to take-off phase which were responsible for generating vertical velocity. It was concluded that there was evidence for mechanical, biomechanical and muscular mechanisms. The former relates to the generation of vertical velocity by the body riding over the base of support; the second is the elastic re-utilization of energy; and the third is the contribution by concentric muscular contraction. The increase in the long jump distance is determined by three basic elements: horizontal velocity, vertical velocity and height of the center of gravity during the flight. the role of muscle strength as a basic variable in jumping is already proven. What is added in this study is the importance of some other physical and kinetic variables such as $30 \mathrm{~m}$ sprint, vertical jump, horizontal velocity at take-off, and long jump completion. It seems that the deep jump (vertical - horizontal) exercises are more effective for physical capacity than kinetic variables.

\section{Conclusions}

The exercises using varied jumps in the style of deep jump (horizontal - vertical) are effective in the development of muscular capacity of long jump athletes. Furthermore, this training method is helpful in sport performance measures like speed and strength which is resembled in the results of $30 \mathrm{~m}$ sprint, vertical jump, horizontal velocity at take-off, and long jump completion tests in the present study. Therefore, vertical and horizontal plyometric exercises are effective alternative to traditional plyometric training.

\section{Funding}

This research received no external funding.

\section{Conflicts of Interest}

The authors declare no conflict of interest. 


\section{References}

1. Pavlovic R, Bonacin D, Stankovic D. Differences in Kinematic Parameters of the Long Jump between Male and Female Finalists of World Championships-Berlin 2009. International Journal of Science Culture and Sport, 2016; 4(4): 353- 366. https://doi.org/10.14486/IntJSCS528

2. Smajlović N. Athletics/textbook. Sarajevo: Faculty of Sport and Physical Education; 2010.

3. Pavlović R. Athletics. Niš: Writers' Association Branko Miljković; 2016.

4. Jaitner T, Mendoza L, Schöllhorn. Analysis of the long jump technique in the transition from approach to takeoff based on time-continuous kinematic data. European Journal of Sport Science, 2001; 1(5): 1- 12. https://doi.org/10.1080/17461390100071506

5. Slawinski J, Termoz N, Rabita G, Guilhem G, Dorel S, Morin JB, et al. How 100-m event analyses improve our understanding of world-class men's and women's sprint performance. Scand J Med Sci Sports, 2017; 27: 45- 54. https://doi.org/10.1111/sms.12627

6. Morin JB, Bourdin M, Edouard P, Peyrot N, Samozino P, Lacour JR. Mechanical determinants of 100-m sprint running performance. Eur J Appl Physiol, 2012; 112: 3921- 3930. https://doi.org/10.1007/s00421-012-2379-8

7. Mero A, Komi PV, Gregor RJ. Biomechanics of sprint running. A review. Sports Med, 1992; 13: 376- 392. https://doi.org/10.2165/00007256-199213060-00002

8. Loturco I, Pereira LA, Cal Abad CC, D'Angelo RA, Fernandes $\mathrm{V}$, Kitamura K, et al. Vertical and horizontal jump tests are strongly associated with competitive performance in 100-m dash events. J Strength Cond Res, 2015; 29: 1966- 1971. https://doi.org/10.1519/JSC.0000000000000849

9. Lees A, Fowler N, Derby D. Abiomechanical analysis of the last stride, touch-down and take-off characteristics of the women's long jump. Journal of Sports Sciences, 1993; 11(4): 303-314. https://doi.org/10.1080/02640419308730000

10.Pavlović R. The morphological status of the finalist in jumping disciplines at the Beijing Olympics. SPORT SCIENCE- International Scientific Journal on Kinesiology, 2012; 5(2): 43-48.

11.Judge LW. Developing Speed Strength: In-Season Training Program for the Collegiate Thrower. Strength and Conditioning Journal, 2007;29:42-54. https://doi.org/10.1519/00126548-200710000-00007

12.Contreras B, Vigotsky AD, Schoenfeld BJ, Beardsley C, McMaster DT, Reyneke JH, et al. Effects of a Six-Week Hip Thrust vs. Front Squat Resistance Training Program on Performance in Adolescent Males: A Randomized Controlled Trial. J Strength Cond Res. 2017; 31: 999-1008. https://doi.org/10.1519/JSC.0000000000001510

13.Kale M, Asci A, Bayrak C, Acikada C. Relationships among jumping performances and sprint parameters during maximum speed phase in sprinters. $J$ Strength Cond Res, 2009; 23: 2272-2279. https://doi.org/10.1519/JSC.0b013e3181b3e182
14.Loturco I, Kobal R, Kitamura K, Abad CCC, Faust B, Almeida L, et al. Mixed training methods: effects of combining resisted sprints or plyometrics with optimum power loads on sprint and agility performance in professional soccer players. Front Physiol, 2017; 8: 1034. https://doi.org/10.3389/fphys.2017.01034

15.Kamnardsiria T, Janchaia W, Khuwuthyakorna P, Suwansrikhama P, Klaphajoneb J, Suriyachanc P. Knowledge-Based System Framework for Training Long Jump Athletes Using Action Recognition. JAIT 2015:182-93. https://doi.org/10.12720/jait.6.4.182-193

16.Bridgett A, Galloway M, Linthorne NP. The Effect of Run-up Speed on Long Jump Performance. In: Scientific Proceedings of the XXth International Symposium on Biomechanics in Sports, Cáceras, Spain, Caceras: Universidad De Extremadura; 2002. P. 80-83

17.Dobbs CW, Gill ND, Smart DJ, McGuigan MR. Relationship between vertical and horizontal jump variables and muscular performance in athletes. The Journal of Strength \& Conditioning Research, 2015; 29(3):661-71. https://doi.org/10.1519/JSC.0000000000000694

18.Fleck SJ, Kraemer W. Designing resistance training programs, 4E. Human Kinetics; 2014.

19.Faigenbaum AD, McFarland JE, Keiper FB, Tevlin W, Ratamess NA, Kang J, Hoffman JR. Effects of a shortterm plyometric and resistance training program on fitness performance in boys age 12 to 15 years. Journal of sports science \& medicine, 2007;6(4): 519.

20.Sharma SK, Saiyad S, Nandwani R. Effect of Plyometric Training on Long Jump Performance in Athletes. Ind Jour of Physioth and Occupat Therapy - An Inter Jour, 2013;7:181. https://doi.org/10.5958/j.0973-5674.7.2.037

21.Canavan PK, Vescovi JD. Evaluation of power prediction equations: peak vertical jumping power in women. Medicine \& Science in Sports \& Exercise, 2004; 36(9):1589-93. https://doi.org/10.1249/01.MSS.0000139802.96395.AC

22.Kotzamanidis C. Effect of plyometric training on running performance and vertical jumping in prepubertal boys. J Strength Cond Res, 2006; 20(2): 441-445. https://doi.org/10.1519/00124278-200605000-00034

23.Hewett TE, Stroupe AL, Nance TA, Noyes FR. Plyometric training in female athletes: decreased impact forces and increased hamstring torques. The American Journal of Sports Medicine. 1996; 24(6):765-73. https://doi.org/10.1177/036354659602400611

24.Šimek-Šalaj S, Milanovic D, Jukic, I. The effects of proprioceptive training on jumping and agility performance. Kinesiology, 2007; 39(2): 131-141.

25.Marković G, Jukić I, Milanović D, Metikoš D. Effects of sprint and plyometric training on morphological characteristics in physically active men. Kinesiology, 2005; 37(1): 32-39.

26. Sharkey BJ. Physiology of fitness. Kuman kinetics; Chamaign; 1990. 


\section{Information about the authors:}

Amir Vazini Taher; https://orcid.org/0000-0001-7780-7054; vazinitaher@gmail.com; Education Administration, Physical Education and Sport Sciences, Hamadan, Iran.

Ratko Pavlović; http://orcid.org/0000-0002-4007-4595; pavlovicratko@yahoo.com; Faculty of Physical Education and Sport, University of East Sarajevo; East Sarajevo, Bosnia and Herzegovina.

Shahram Ahanjan; https://orcid.org/0000-0003-3047-3266; vazinitaher@gmail.com; Amirkabir University of Technology, Department of Physical Education, Iran.

Iryna Skrypchenko; http://orcid.org/0000-0001-5895-3099; sit71@ukr.net; Dnipropetrovs'k State University of Internal Affairs, Dnipro, Ukraine.

Marko Joksimović; (Corresponding Author); http://orcid.org/0000-0003-4232-5033; nicifor007@outlook.com; Football Club Nacional; Podgorica, Montenegro.

Cite this article as:

Vazini Taher A, Pavlović R, Ahanjan S, Skrypchenko I, Joksimović M. Effects of vertical and horizontal plyometric exercises on explosive capacity and kinetic variables in professional long jump athletes. Pedagogy of Physical Culture and Sports, 2021;25(2):108-113.

https://doi.org/10.15561/26649837.2021.0205

This is an Open Access article distributed under the terms of the Creative Commons Attribution License, which permits unrestricted use, distribution, and reproduction in any medium, provided the original work is properly cited (http://creativecommons.org/licenses/by/4.0/deed.en).

Received: 10.07.2020

Accepted: 25.09.2020; Published: 30.04.2021 